\title{
A Cellular Automata Traffic Flow Model Based on Multi-trigger- headways
}

\author{
Ziyin $\mathrm{Xu}^{1}$, Kechen Wang ${ }^{2}$ \\ ${ }^{1}$ Faculty of Science, Jiangsu University, Zhenjiang, 212000, China \\ ${ }^{2}$ School of Energy and Power Engineering, Jiangsu University, Zhenjiang 212000, China
}

\begin{abstract}
The paper provides a more practical cellular automata traffic flow model for a dual-lane highway traffic system by introducing the rules of emergency braking and gentle braking, along with their trigger headways, and make it available to Intelligent Transportation System. According to the results of computer simulation, the variation trends of average velocity, velocity variance, braking times and overtaking times under different traffic intensities and expected headways are analyzed. An evaluation for the performance of different expected headways in ITS under different traffic intensities is made in order to find out the optimum range.
\end{abstract}

Keywords: Traffic flow; Cellular automata; ITS; Multi-trigger-headway; Energy conservation.

\section{Introduction}

With the rapid growth of the social economy and the increasing number of motor vehicles in the modern era, traffic problems are increasingly impeding the development of the city. Take the highway as an example, as the backbone road connecting important areas, once it becomes congested, it will cause great social repercussions. If highway service level and user satisfaction decrease, it will have a negative impact on the highway management department. Its intelligent traffic application is based on a traffic flow model that can accurately describe the actual road conditions. Existing traffic flow models fall into two categories: macroscopic hydrodynamic models and microscopic cellular automata models [1]. The latter is easy to operate and easy to control the micro rules. Moreover, it meets intelligent traffic demand and is widely used in traffic flow simulation [2-4]. The original cellular automata traffic flow model was the rule 184 model proposed by S. Wolfram in 1983 [5]. Nagel and Schreckenberg extended rule 184 in 1992 and proposed the famous NaSch model [6], which considers the random slowdown process. Later, Wolf et al. [7] proposed the famous STCA (Symmetric two-lane Cellular Automata) model for the limitations of non-overtaking, and successfully introduced the lane change rule.

At present, most of the research is based on single-lane following model for deformation and correction. Deterministic deceleration rules in NaSch force the velocity to be equal to the distance from the vehicle in front to avoid collisions. In practice, however, most drivers do not choose to make emergency braking until they are close to colliding with the front car. Instead, they consider the current distance from the front car and their own velocity to maintain a safe distance. Slow deceleration begins when the distance is less than the safe distance, that is, the driver is more inclined to follow the vehicle in front with the expected distance. Accordingly, Parker [8] first proposed a model based on expected spacing in 1996. However, it is still difficult to quantify the characteristics of road traffic such as emergency braking when only expected headways are used. In this paper, by introducing the emergency headway and visible headway as the trigger condition of the deceleration and overtaking rules, the traffic flow of the highway is described more accurately, and the driving characteristics can be extracted easily. Moreover, the result of the simulation can be easily connected with the energy conservation and emission reduction, which are hot issue recently.

\section{A Cellular Automata Traffic Flow Model Based on Multi-trigger-headways}

Highways are seen as two parallel discrete cellular chains. Its length is L, and every cell can be occupied by at most one car at any moment. The left and right lanes are defined as lane 1 and lane 2, respectively. It is assumed that the driving road of the vehicle is an ideal flat road. In the driving 
process of the vehicle, there is only random change of velocity along the driving direction, but no sideslip movement, that is, the driving direction of the vehicle is always forward. According to the traffic characteristics of China's highways, compact cars (fast cars) and large trucks (slow cars) are the main vehicle types, and the ratio of slow cars is recorded as r. Fast lanes (lane 1) allow only compact cars, slow lanes (lane 2) allow large trucks and compact cars to pass. The maximum and minimum velocity limit of lane $\mathrm{j}$ are denoted as $V_{\max }^{j}$ and $V_{\min }^{j}$. The velocity may be below the minimum velocity limit only when the highway becomes congested. Compact cars can use the left lane to overtake the front car, changing the lane to overtake when accelerating. Due to the fast velocity of the compact cars passing the lane, the time consumed in changing the lane of the vehicles can be ignored. Now the truck is defined as vehicle type 1, the compact car in the non-overtaking state is defined as type 2, and the compact car in the overtaking process is defined as type 3 . The expected velocity (i.e. maximum velocity) of vehicle type $\mathrm{i}$ is denoted as $V_{E}^{i}$. Then, at time $\mathrm{t}$, the distance between the kth car and the preceding car on lane $\mathrm{j}$ can be expressed as:

$$
d_{k}^{j}(t)=\frac{l_{k+1}^{j}(t+1)-l_{k}^{j}(t)-1}{v_{k}^{j}(t)}
$$

where $\mathrm{l}_{\mathrm{k}}^{\mathrm{j}}(\mathrm{t})$ and $\mathrm{v}_{\mathrm{k}}^{\mathrm{j}}(\mathrm{t})$ represents the location and velocity of $\mathrm{kth}$ car on lane $\mathrm{j}$ at time $\mathrm{t}$.

\subsection{Single-lane Following Model with Multiple Trigger Headways}

In the previous single-lane following model, the mandatory deceleration rule in NaSch is often used. When the distance between the vehicle and the vehicle in front is less than the current velocity, the velocity is forced to be equal to the distance between the vehicle and the vehicle in front to avoid collision. That is, when $v_{k}^{j}(t)>l_{k+1}^{j}(t+1)-l_{k}^{j}(t)-1$, setting $v_{k}^{j}(t)=(t+1)-l_{k}^{j}(t)-1$.In this paper, deterministic deceleration is divided into emergency braking and gentle braking, and their trigger headways are defined as the emergent headway $D_{0}$ and the expected headway $D_{E}$ respectively. The vehicle update rules in the one-lane following model are as follows:

(1). Emergency braking rule

In order to avoid the front and rear car collision, sufficient emergency braking time must be reserved to prevent the accident. This headway is defined as the emergent headway $D_{0}$. Due to the different psychological quality of drivers, it is assumed that the emergent headway follows normal distribution. When the headway of the current car is less than $D_{0}$, emergency braking shall be adopted to ensure that there is no collision with the front car, that is, when

$$
d_{k}^{j}(t)<D_{0}
$$

Setting:

$$
v_{k}^{j}(t+1)=\left\lfloor\frac{l_{k+1}^{j}(t)-l_{k}^{j}(t)+1}{D_{0}}\right\rfloor
$$

(2). Gentle braking rule

Drivers always want to keep a certain distance with the vehicle in front, which is usually larger than the emergent headway and varies from person to person. This headway is defined as the expected headway $D_{E}$. Due to the differences in personality and mentality of each driver, it is assumed that the expected headway is subject to normal distribution. When the time headway of the current vehicle is less than $D_{E}$, the driver will take gentle braking and keep the appropriate distance, that is, when:

$$
d_{k}^{j}(t)<D_{E}
$$

Setting: 


$$
v_{k}^{j}(t+1)=v_{k}^{j}(t)-1
$$

(3). Accelerating rule

When there is no need to take deterministic deceleration (emergency braking and gentle braking), the vehicle is expected to reach the maximum velocity of the vehicle, i.e

$$
v_{k}^{j}(t+1)=\min \left(v_{k}^{j}(t)+1, V_{E}^{i}\right)
$$

(4). Random slowdown rules

Drivers often slow down slightly during driving because of blurred vision, but not enough to go below the minimum velocity limit. This process can be characterized by the random slowdown rate $p_{s}$, that is, when $v_{k}^{j}(t)>V_{\text {min }}^{j}$, the probability of $p_{s}$ leads to:

$$
v_{k}^{j}(t+1)=\max \left(v_{k}^{j}(t)-1, V_{\min }^{j}\right)
$$

In addition, when emergency braking has been initiated, random slowdowns are no longer performed.

\subsection{Rules for Changing Lanes on Dual-lane}

In the actual situation, the vehicle cannot change the lane to overtake at will, and the distance of the vehicle before and after the side lane should be paid attention to. At this point, the maximum distance between the car and the car before and after the side lane is defined as the lane-change headway $D_{L}$, which is generally equal to the driver's expected headway $D_{E}$. In addition, the vehicle cannot overtake when it is too far away from the vehicle in front, or it may overtake without seeing the vehicle in front, which does not conform to the reality. When the overtaking is triggered, the shortest distance from the front car is defined as visible headway $D_{P}$, which is normally distributed. There is a probability of $p_{l}$ for a fast car under non-emergency to overtake the slow car in front when the condition of lane-change headway and visible headway are met.

\section{Simulation Analysis of the Model}

In the numerical simulation, the classical cellular length is $7.5 \mathrm{~m}$. Take the length of the highway as $7.5 \mathrm{~km}$, that is, the road is divided into 1,000 cells. Since the arrival of vehicles is random, the mutual influence is weak, and the external factors do not interfere, the intensity of imported traffic is set as $\mathrm{q}(\mathrm{vel} / \mathrm{s})$. The exit boundary conditions adopt pseudo open boundary conditions. That is, when the vehicle goes to the exit, the position of the next time step car is judged. If it goes beyond the road boundary, it will be shifted to the road tail cell, which ensures that every vehicle will pass the road tail cell. The traffic flow can be obtained by calculating the number of vehicles through the road tail cell, which is convenient for data extraction. The maximum and minimum velocity of the slow lane is $4(\mathrm{cell} / \mathrm{s})$ and 3(cell/s), respectively; Fast lane maximum and minimum velocity are 5(cell/s) and 4(cell/s); The expected velocity of the slow car, non-overtaking fast car and overtaking fast car is 3 , 4 and 5, respectively. In non-intelligent traffic, the expectation of the emergent headway is $1.25 \mathrm{~s}$ and the variance is $0.04 \mathrm{~s}^{2}$. The variance of expected headway is $0.64 \mathrm{~s}^{2}$. The expectation of visible headway is $4.5 \mathrm{~s}$ and the variance is $1 \mathrm{~s}^{2}$. The random slowdown rate is 0.3 . The probability of overtaking is 0.9. In intelligent traffic, all the trigger headway expectation is unchanged and the variance is set to 0 . The random slowdown rate is 0 . The probability of overtaking is 1 . Each iteration of the system takes 72,000 steps, leaving out the first 2,000 steps to eliminate transient effects. 


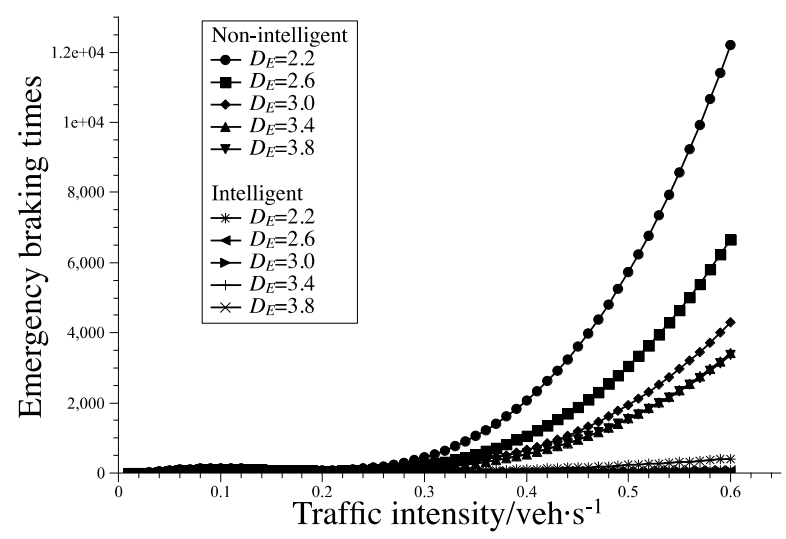

Figure 1 . The relation between emergency braking times and traffic intensity

As we can see from figure 1, in the case of on-intelligent traffic system, when the traffic intensity $\mathrm{q}$ of is less than $0.3 \mathrm{veh} / \mathrm{s}$, the value of expected headway has little influence on the number of emergency braking. In this stage, the changes of emergency braking times with the traffic intensity can be almost ignored. When the intensity $\mathrm{q}>0.3 \mathrm{veh} / \mathrm{s}$ of traffic flow increases, the number of emergency braking decreases significantly with the increase of expected headway. It is worth noting that when the expected headway $\mathrm{D}_{\mathrm{E}}$ is greater than 3.4, the number of emergency braking is nearly unchanged. In fact, with the increase of expected headway, the driver always wants to keep a larger distance from the vehicle in front, and the space between vehicles is not easy to reach the emergency headway, so the emergency braking times are significantly reduced. However, this effect only occurs when the expected headway has not increased to a certain extent. When the expected headway is already large, that is, when the distance between the driver and the vehicle in front has been maintained large enough, the effect of further increasing the headway on the emergency braking times of the vehicle becomes negligible. In intelligent traffic system, the brake times change little with the traffic intensity and the expected headway, and remain at a very low level.

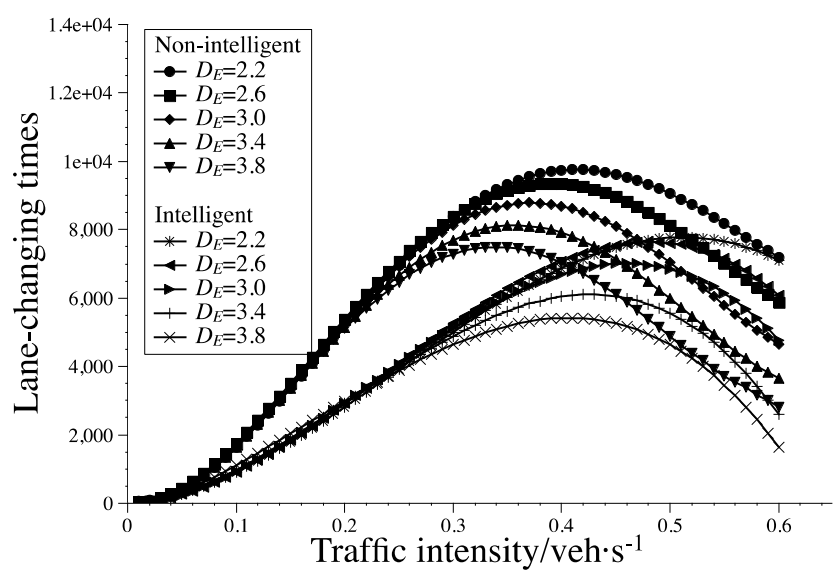

Figure 2. The relationship between lane change times and traffic intensity

FIG. 2 shows the relationship between different traffic intensities and different expected headways and the total number of road lane changes in the intelligent and non-intelligent cases respectively. It can be seen from figure 2 that, in the case of non-intelligent traffic system, when the intensity $q$ is less than $0.2 \mathrm{veh} / \mathrm{s}$, the value of expected headway has little influence on the number of emergency braking. However, in this stage, the number of changing lanes grows rapidly with the strength of traffic flow, and the slope of the curve is very large. When the traffic intensity $q>0.2 \mathrm{veh} / \mathrm{s}$, the value of expected headway gradually increases, but the number of changing lanes starts to increase and then decrease with the increase of the strength of traffic flow. It is worth noting that, with the increase of the expected headway value, the peak point of the switching frequency shifts towards the direction 
of the traffic intensity being smaller. That is, under the same traffic intensity, the value of the switching frequency is inversely proportional to the value of the expected headway. In fact, when the driver changes the lane, he should consider the distance between the front and rear vehicles in the adjacent lane. When the expected headway is larger, the driver wants to maintain a larger headway between the front and rear vehicles after the change of lane. Therefore, the condition of changing the lane becomes harsher, and the number of changing lanes is reduced. In the case of intelligent traffic, when $\mathrm{q}<0.25 \mathrm{veh} / \mathrm{s}$, the value of expected headway also has little impact on the number of emergency braking, but the number of lane change increases slowly with the traffic intensity in this stage. When the traffic intensity $q>0.25 \mathrm{veh} / \mathrm{s}$, the peak point of the number of lane changes was shifted to the right as a whole compared with that of non-intelligent. The number of lane changes has both good and bad effects on traffic. Fewer times of lane changes can improve road safety, and the vehicles may be in a more reasonable driving state. On the other hand, a less frequent change of lanes may result in an express train following a slow train but being unable to change lanes. It can be seen from figure 3 and 4 that the average velocity size and stability of both fast lane and slow lane are at a higher level under intelligent traffic. Therefore, the reduction of lane change at this place has a positive impact on traffic flow. It is worth noting that when the expected headway is higher, the average velocity of the slow lane in the intelligent system drops sharply with the increase of traffic intensity. Thus, in the intelligent control, the expected headway should not be too large.

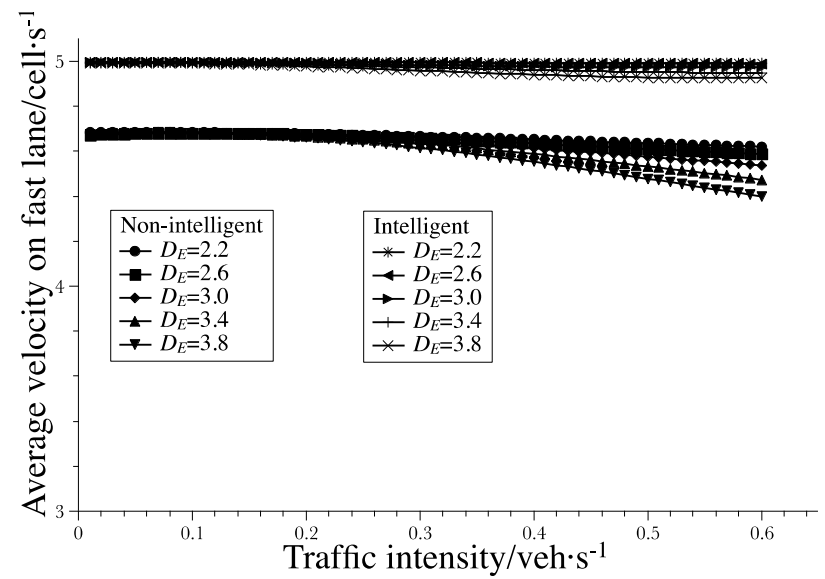

Figure 3. The relation between average velocity of fast lane and traffic intensity

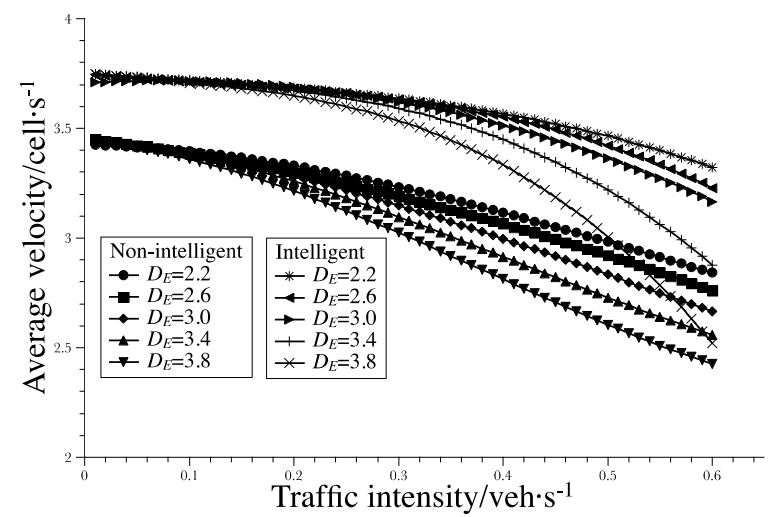

Figure 4. The relation between average velocity of slow lane and traffic intensity

As can be seen from figure 5, when the value of expected time span is $\mathrm{D}_{\mathrm{E}}<2.3 \mathrm{~s}$, the number of emergency braking decreases sharply with the increase of expected time span. When the expected headway is $D_{E}>2.5 \mathrm{~s}$, the number of emergency braking only increases slowly. Therefore, the headway of $D_{E}<2.3 \mathrm{~s}$ should be avoided when the expected headway is evaluated. 


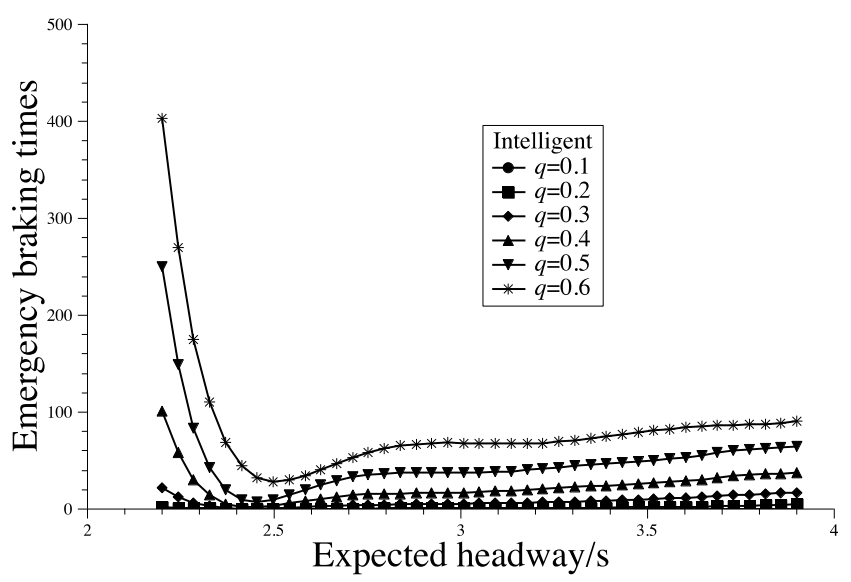

Figure 5. Relation between emergency braking times and expected headway

Figure 6 shows the relation between different traffic intensities, different expected headways and the total number of road lane changes under intelligent traffic. Under low traffic intensity, lane change is less frequent and less affected by the value of expected headway. In fact, when road traffic intensity is low $(\mathrm{q}<0.3 \mathrm{veh} / \mathrm{s})$, due to the small number of vehicles on the road and relatively weak interaction between vehicles, traffic flow can be regarded as free flow, and drivers rarely need to change lanes.

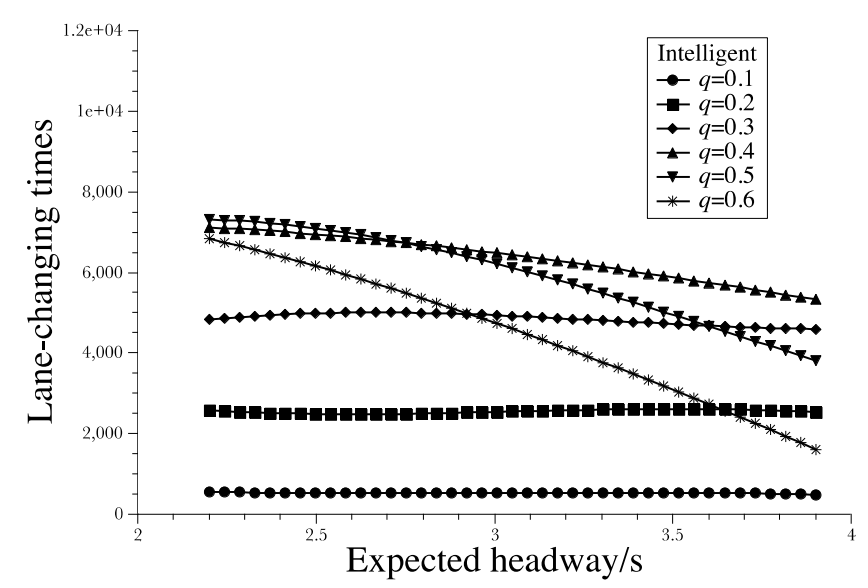

Figure 6. The relationship between the lane changing times and expected headway

In addition, the paper also analyzed the score results under intelligent traffic, taking into account the number of road change, the number of emergency braking and the average velocity of different expected headways, and the traffic intensity. According to the analysis, the curve curvature was relatively steep before the peak point appeared, indicating that the comprehensive traffic flow score was sensitive to the value of expected headway. The peak point indicated the current expected headway between $D_{E}=2.4 \mathrm{~s}-2.5 \mathrm{~s}$, which was smaller than the three-second headway stipulated by the state, and the road utilization rate was higher. It is worth noting that the lower the traffic intensity, the more the traffic flow can be regarded as free flow, and the smaller the influence of the value of expected headway on the fraction.

Figure 7 shows the relation between different traffic intensity, different expectation headway and road velocity standard deviation in the intelligent and non-intelligent cases respectively. It can be clearly seen from figure 7 that the velocity standard deviation of intelligent traffic is significantly lower than that of non-intelligent traffic. Moreover, with the increase of the traffic intensity, the standard deviation of the average velocity of vehicles increases, and the traffic unevenness increases. In fact, from a safety perspective, the driver always wants the vehicle to be driven at the same velocity on the road, that is, the smaller the standard deviation of the velocity, the better. Therefore, when the 
density of traffic flow increases, a better expected headway value plays an important role in the safety of traffic flow.

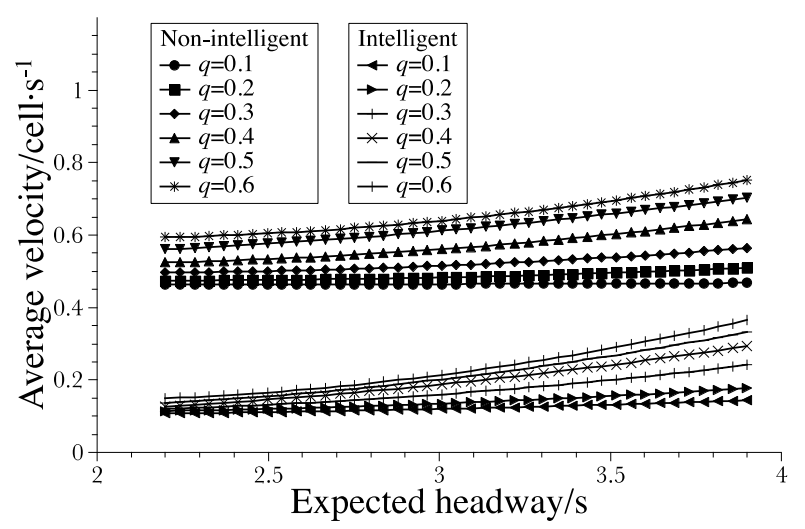

Figure 7. The relationship between variance and expected headway

\section{Summary}

In this paper, the existing micro traffic flow model is analyzed, and a cellular automata traffic flow model based on multi-trigger headway is proposed. In this paper, two kinds of time headway of trigger braking are introduced innovatively, which are defined as the emergent headway and the expected time headway respectively, which make up for the defects of the existing model. The research results have the following significance:

(1). This model takes into account the different driving characteristics of vehicles in the process of acceleration, deceleration and lane change caused by human factors. For each driver, the switching probability, expected headway and control of the trigger headway are normally distributed. It restores the real road condition of the highway and provides a real traffic flow model for the realization of intelligent traffic.

(2). The cellular automata traffic flow model based on multi-trigger headway is extended to be intelligent. According to the simulation results, under intelligent traffic mode, the number of vehicles changing lanes and emergency braking is significantly reduced, and the vehicle velocity distribution and average velocity are significantly increased, revealing the potential apply in energy conservation and emission reduction.

(3). In the intelligent mode of highway, taking road capacity, emergency braking times, lane change times and average velocity into account, the optimal headway is obtained by evaluating the value of different expected headway and the traffic intensity.

(4). This model has strong practicability. It can change the parameters according to different application backgrounds, and the data can be extracted easily, providing more accurate simulation and optimization methods for intelligent driving decision-making.

\section{References}

[1]. WANG Yongming, ZHOU Leishan, LU Yongbo. Vehicle-changing rule based on cellular automata traffic flow model [J]. China journal of highway, 2008, 21(1):89-93.

[2]. MAO Yongmao, ZHONG Chengwen. Cellular automata traffic flow model based on safe driving [J]. Journal of physics, 2005, 54(12):5597-5601.

[3]. DONG Liyun, XUE Yu, DAI Shiqiang. A one-dimensional cellular automata traffic flow model based on the heel car idea [J]. Applied mathematics and mechanics, 2002, 23(4):331-337.

[4]. S. Wolfram. Statistical mechanics of cellular automaton[J]. Rew. Mod. Phys, 1983, 55(3):601644. 
[5]. K. Nagel, M. Schreckenberg. A cellular automaton model for freeway traffic[J]. J. Phys. I.France, 1992, 2:2221-2229.

[6]. Chowdhury D, Wolf D E, Schreckenberg M. Particle hopping models for two-lane traffic with two kinds of vehicles: Effects of lane-changing rules[J]. Physica A Statistical Mechanics \& Its Applications, 1997, 235(3-4):417-439.

[7]. Parker M T. Effect of heavy goods vehicles and following behaviour on capacity at motorway roadwork sites[J]. Traffic Engineering \& Control, 1996, 37(9):524-531.

[8]. YUAN Haiying, YANG Xiaokuan, ZHANG Jinxi, et al. Study on the running velocity model in the free flow state of highway [J]. Traffic transportation research, 2005(5):73-75. 